\title{
Pecularities of pore relaxation in nanoclusters
}

\author{
M.A.Ratner ${ }^{1}$, V.V.Yanovsky ${ }^{1,2}$ \\ ${ }^{1}$ Institute for Single Crystals, STC "Institute for Single Crystals", \\ National Academy of Sciences of Ukraine, \\ 60 Nauky Ave., 61001 Kharkiv, Ukraine \\ ${ }^{2}$ V.Karazin Kharkiv National University, \\ 4 Svobody Sq., 61022 Kharkiv, Ukraine
}

\section{Received September 10, 2016}

\begin{abstract}
Process of the relaxation of a cluster with an intrinsic pore was investigated for different phases of a cluster. Main parameters, determining pore dissolving time were found. In particular, it was shown that there exists critical temperature $T_{c}$, that depends on pore and cluster sizes. Below $T_{c}$ nonequilibrium process does not destroy solid state of the cluster and pore life time increases sharply. Process of pore relaxation in this case is characterized by a free energy barrier.

Keywords: cluster, phase state, pore, nonequilibrium process, relaxation.
\end{abstract}

Исследован процесс релаксации кластера с внутренней порой для различных агрегатных состояний кластера. Найдены основные параметры, определяющие время растворения поры. В частности, показано, что существует критическая температура $T_{c}$, зависящая от формы и размера кластера. При начальных температурах кластера ниже $T_{c}$, неравновесный процесс не разрушает твёрдое состояние кластера и время жизни поры резко возрастает. Процесс релаксации поры в этом случае характеризуется наличием барьера свободной энергии.

Особливості релаксації пори у нанокластерах. М.А.Ратнер, В.В.Яновський.

Досліджено процес релаксації кластера з внутрішньою порою для різних агрегатних станів кластера. Знайдено основні параметри, що визначають час розчинення пори. Зокрема, показано, що існує критична температура $T_{c}$, залежна від розмірів пори та кластера. При початкових температурах кластера нижче $T_{c}$ нерівноважний процес не порушує твердого стану кластера та час життя пори різко збільшується. Процес релаксації пори у цьому випадку характеризується наявністю бар’єра вільної енергії.

\section{Introduction}

It is well known that properties of materials are at large degree determined by their defect structure. Various defects of materials can be classified by their dimensionality. Thus, pores and new phase inclusions relate to 3-dimensional defects, while grain boundaries relate to 2-dimensional and dislocations to 1-dimensional ones. It is naturally to classify such elementary objects as vacancies and atoms in interstitials as 0 -dimensional defects. If estimated according to the effect on many material properties, 3-dimensional defects, such as pores and new phase inclusions, play the key part. In the bulk materials, where the role of boundaries is insignificant, theory of behavior and evolution of such objects is well developed (see, e.g., [1]). However, in connection with intensive investigation of nanomaterials, where the influence of boundaries is enormous, it occurred, that, in such materials, the behavior of pores and new phase inclusions is not sufficiently investigated. Thus, in the present work, the attempt is made to investigate relaxation of 
vacancy pores in nanocrystal clusters with pair interactions of atoms. For modeling such clusters, their unusual properties play a key part. In particular, let us point out some special features concerning with phase states of small clusters, that were investigated, particularly, in [2, 3]. For such clusters phase transitions are connected not only with heat motion of atoms in a cluster. Thus, in clusters of small sizes, following phase states are marked out: liquid, solid and the one with a solid core and liquid shell. Relatively high electron excitation energies of a solid state cluster b correspond to configuration change of cluster atoms. Liquid state is considered to be the highest configuration excitation. There exist a large number of free energy local minima of a cluster at finite temperature. Each of these minima corresponds to a different configuration of cluster atoms. One of them corresponds to a stable state while the rest relate to metastable ones. Thus, one of the type of configuration excitation is considered to be the going of atoms out cluster core to its surface, that diminishes the total interaction energy of cluster atoms but increases system configuration entropy.

In the works [4, 5], for clusters of small sizes, it was demonstrated numerically, that high configuration excitations of a cluster correspond to the formation of cavities inside it (at low excitations the vacancies arise). At transition of a cluster from a liquid state to solid one, the cavities go out of the cluster. Such process, related to diffusion of cavities towards cluster surface, is of activation (barrier) character.

\section{Model and method}

\subsection{Model of nanoparticle(cluster) with} an inside pore

Initially, atoms with the mass $m=39.9$ a.u. in the nanoparticle formed a cubic lattice with interatomic distance $a=4.816 \AA$. In the model, the number of atoms $N$ was chosen equal to 64 and 512. Atoms were interacting according to Lennard-Jones potential with the following parameters, taken for Argon atoms: $\sigma=3.405 \AA, D=0.0104 \mathrm{eV}$. Here $D$ is the depth of the potential well, $\sigma$ is the finite distance at which the interparticle potential is zero, and $r_{\min }$ is the distance at which the potential reaches its minimum. At $r_{\text {min }}$, the potential function has the value $-\mathrm{D}$. The distances are related as $r_{\text {min }}=21 / 6 \sigma \approx 1.122 \sigma$. Cluster tempera-

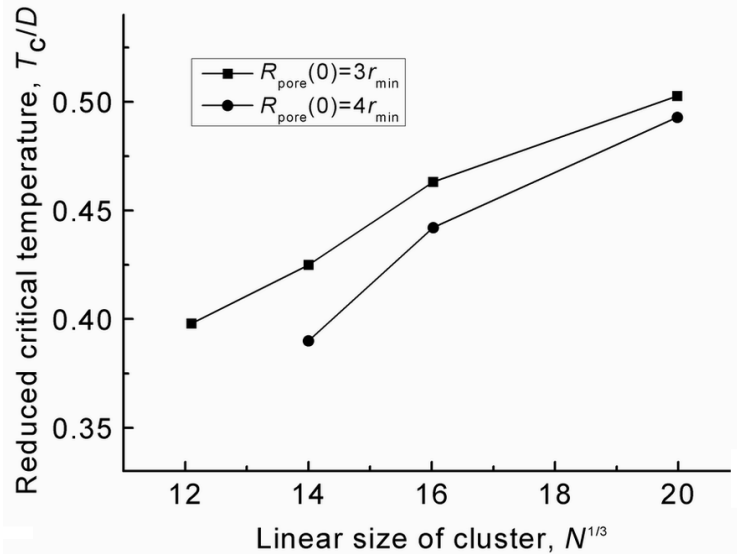

Fig. 1. Dependences of critical temperature, $T_{c}$, on linear size of cluster. Square denote the case of $R_{\text {pore }}(0)=3 r_{\text {min }}$ while circles relate to $R_{\text {pore }}(0)=4 r_{\text {min }}$.

ture $T$ is defined as mean kinetic energy per one atom.

The equations of motion are solved numerically via velocity Verlet algorithm [6$8]$ with a time step $d t=10^{-6} \tau$. The temperatures, chosen for modeling, are lower than melting temperature of Argon $T=83.4 \mathrm{~K}$, which provides for nanoparticle stability (see Fig. 1).

For the nanoparticle with a given $N$, the number $Q=25$ of random realizations is created with the given temperature $T$. After equilibration at the given temperature, a pore of the given initial pore radius $R_{\text {pore }}(0)$ is instantly cut off in the center of a nanoparticle (cluster). After that, the numerical solving of motion equations is conducted by the described above method.

The characterization of pore relaxation is realized in a following way. The whale cluster is overlaid with a three-dimensional cubic lattice with a period $a_{\text {grid }}=0.5 r_{\text {min }}$. A pore is defined as any connected domain of cells, that are free from cluster atoms, with a diameter no less the $1.5 r_{\text {min }}$, and surrounded on all sides by occupied cells. If such domain has a diameter less than 1.5 $r_{\text {min }}$ it is defined as vacancy. Such criterion was obtained in empirical way in order to discern pore with single vacancies and avoid the influence of surface convolution. Cluster a volume is defined as a summarized volume of occupied cells and free cells, belonging to pores or vacancies. In the present work, the temperature range was investigated from 30 to $55 \mathrm{~K}$, clusters, containing from $12^{3}$ to $20^{3}$ atoms with pores spherical pores 
of initial radii $R_{\text {pore }}(0)=3 r_{\min }$ and $4 r_{m i n}$, with initial central position in a cluster.

\subsection{Preparation of initial cluster confor-} mations

In the present work, pore relaxation in the clusters in each of three described in [2] phase states was described: solid, liquid and the one with a solid core and liquid shell (that, in its turn, consists of the set of states with different degrees of ordering [3]).

For characterization of the cluster phase state, the fluctuation $\delta$ was used of square root of mean square distance between neighbor atoms, $a$. According to the conventional criterion [2], for solid state cluster $\delta$ does not exceed $15 \%$ of $a$, for the state with solid core and liquid surface, $\delta$ increases sharply from cluster center to its surface, while for liquid state $\delta$ essentially exceeds $15 \%$ of a throughout the entire cluster.

The time between phase transitions of cluster, in the researched in the given work range of size and temperatures, is too large to be observed during numerical count. In the present investigation, clusters in solid state are obtained in the following way. Initial state of a cluster, constructed via procedure described in Sec. 2.1 is far from equilibrium and, if allowed to relax freely, finishes in liquid state with high enough temperature. Thus, in order to obtain solid state, after assigning initial atom coordinates, cluster is quickly cooled to the given temperature $T$ (that is less than melting temperature). This way, cluster in a liquid state is always obtained, since the system has not enough time to relax to the solid state, even if it is equilibrium. Then, cluster is continued to be cooled down by quasiadiabatic method to temperature $T_{\min }$, that is close to zero, after which is heated by the same quasi-adiabatic procedure for the starting temperature $T$. This way we obtain a cluster in solid state with higher binding energy of atoms, $U_{\text {bond }}$, then a cluster with the same temperature in a liquid state. Entropy difference between liquid and solid phases can be estimated during numerical cal culations as

$$
\Delta S \cong \sum_{T \downarrow}^{T_{\min }} \frac{\Delta Q}{T}+\sum_{T_{\min }}^{T \uparrow} \frac{\Delta Q}{T} .
$$

If, in the described above procedure, $T_{\text {min }}$ is increased, intermediate phase states of a cluster are obtained, corresponding to local minima of its free energy, while $U_{b o n d}$ is the smaller and $\delta$ is the larger, the higher is $T_{\text {min }}$.

Cluster volume diminishes by approximately $4 \%$ and binding energy of atoms increases by 7-9\% after transfer from liquid to solid state. Also, the specific property of solid state is appearance of "breathing mode" of cluster oscillations and increasing pore relaxation time more than by an order of magnitude. The last is connected with the slowing of vacancy diffusion.

Characteristic difference of temperatures between liquid and solid states, obtained from (1), together with known binding energies of atoms in the cluster, gives a theoretical possibility to estimate which state (solid or liquid) is stable for each investigated pair of cluster temperature and size values.

Here it is important to point out the following circumstance. The instant "cutting" pore out of the cluster gives rise to a nonequilibrium process, besides, the deviation of the system from equilibrium is quite large, since pore size is comparable to that of the system. So that, it is natural to suppose that the very event of "cutting off" the pore will destroy more ordered metastable states in favor of more stable less ordered ones (if such exist). Thus, it is natural to start the investigation from the most ordered state, and, while increasing gradually cluster temperature, one can hope to obtain the right statistical picture.

Let us underline that the "inverse" transfer from the liquid state to the solid one as a result of system deviation from equilibrium is little probable since liquid states possess much greater configuration entropy.

Thus, initial cluster conformations in the present work are prepared in the following way. Starting from solid phase conformations at low temperatures (approximately $30 \mathrm{~K}$ ), obtained in 2.1 we increase temperature gradually by $2 \%$ every $0.1 \mathrm{ps}$. This way we obtain solid phase conformations at higher temperatures. Conformations at selected for numerical computations temperatures are relaxing during $5 \mathrm{ps}$, then, by the method described in 2.1, the MD run is started at a constant temperature. Every 2 ps one conformation is fixed that is included in the collection of 25 random conformations at the given temperature.

Below, the temperature will be measured in the units of potential depth $D$ and linear sizes in the units of equilibrium interatomic distance $r_{\min }$ (see item 2.1). 




Fig. 2. Example of pore relaxation a temperature below $T_{c}\left(T=0.42 \mathrm{D}, N=14^{3}, R_{\text {pore }}(0)=\right.$ $3 r_{\text {min }}$ ). Thin lines demonstrate dependence of reduced pore volume on time for first 12 (of total 25) initial cluster random realizations, bold line - averaged dependence (over 25 random realizations).

\section{Results and discussion}

\subsection{Relaxation of pore in a solid cluster}

When initial state of cluster is solid, for each pair of values, $R_{\text {pore }}(0), N$ there exists critical temperature $T_{c}$, below which the nonequilibrium process initiated by cutting a pore off a cluster, does not destroy solid state of the cluster. In this case, pore exists, on average, longer than 12 ps (that is MD simulation time in the given work). The dependence of $T_{c}$ on $N$ for $R_{\text {pore }}(0)=3 r_{\text {min }}$ and $R_{\text {pore }}(0)=4 r_{\text {min }}$ is shown in Fig. 1 . It can be seen from the figure that increase of initial pore radius from $3 r_{\text {min }}$ to $4 r_{\text {min }}$ leads to the lowering of $T_{c}$. Evidently, this is due to the fact that the larger is $R_{\text {pore }}(0)$ the stronger is initial system deviation from equilibrium.

At $T<T_{c}$ each random realization of the process is characterized by a barrier character of dependence $V_{\text {pore }}(t)$. During first moments (about $0.1 \mathrm{ps}$ ) the process is highly nonequilibrium and pore volume diminishes fast. Then process of pore dissolving be comes much slower, until at some, depending on realization number, time moment, the dissolving of pore is essentially accelerated (see examples for $N=14^{3}, R_{\text {pore }}(0)=$ $3 r_{\text {min }}$ and $R_{\text {pore }}(0)=4 r_{\text {min }}$ in Figs. 2,3). Approximately for half of random realizations, $V_{\text {pore }}$ becomes independent on time (in the observed time interval $12 \mathrm{ps}$ ). It is interesting, that for different random realizations $V_{\text {pore }}(t)$ is stabilized at the same values.

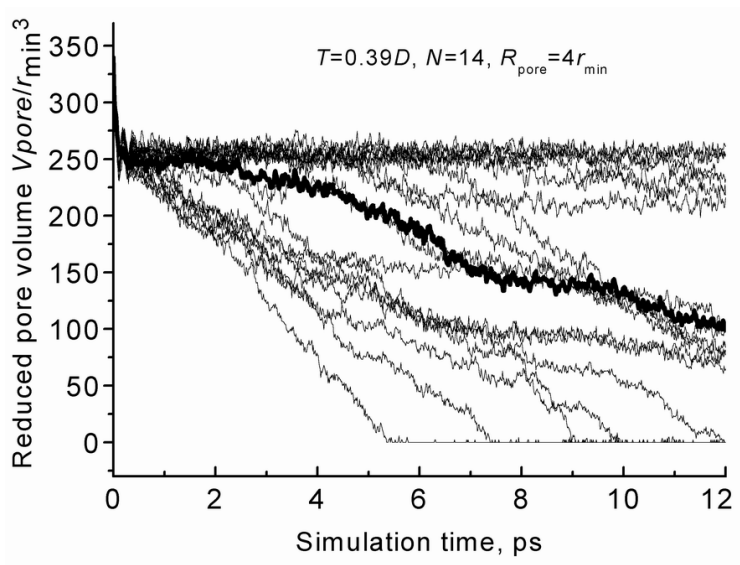

Fig. 3. Example of pore relaxation a temperature below $T_{c}\left(T=0.42 \mathrm{D}, N=14^{3}, R_{\text {pore }}(0)\right.$ $\left.=4 r_{\text {min }}\right)$. Thin lines demonstrate dependence of reduced pore volume on time for first 12 (of total 25) initial cluster random realizations, bold line - averaged dependence (over 25 random realizations).

Explanation of the obtained dependence $V_{\text {pore }}(t)$ could be found in the results presented in the works [4,5], where it is shown numerically that high configurational excitation of a cluster correspond to the formation of voids in its interior. (These voids can be associated with the artificially created pore in our investigation.) Transition of the cluster to a lower state is accompanied by the moving of voids out of the cluster. Such process of transition between different states requires overcoming a free energy barrier.

From the look of dependences $V_{\text {pore }}(t)$ for $T<<T_{c}$, one can make a conclusion of an existence of certain values of "preferred by system" pore volume $V_{\text {opt }}$, corresponding to excited configurational states and separated from the basic state by free energy barriers. Thus, for $N=14^{3}, R_{\text {pore }}(0)=3$ $r_{\text {min }}$, the observed values $V_{\text {opt }} \sim 65 r_{\min }{ }^{3}$ and $90 r_{\min }{ }^{3}$, for $R_{\text {pore }}(0)=4 r_{\text {min }}$, the observed values $V_{\text {opt }} \sim 260 r_{\min }{ }^{3}$. It is worth to note, that "optimal" values $V_{\text {opt }}$ of pore volume weakly depend on initial cluster size but increase sharply with the growth of initial pore volume (see examples in Figs. 2, 3).

Thus, process of relaxation of cluster with a pore inside, consists in transition from the excited configurational state into the lower one and is of barrier character.

Let us underline, that for considered case $T<T_{c}$, the final state of a cluster remains solid, and pore, until it has been dissolved, retains connectivity and central position in the cluster. Thus, we observe the 


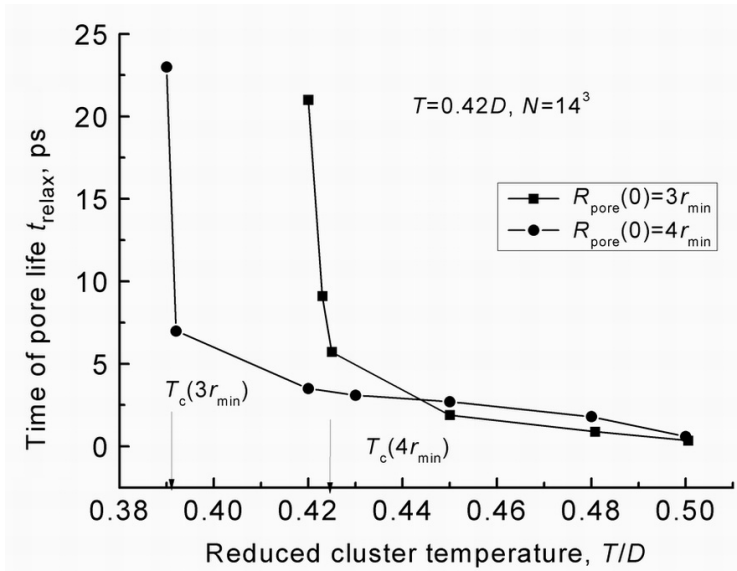

Fig. 4. Dependences of pore life time on cluster temperature while heating cluster starting from solid state. Square denote the case of $R_{\text {pore }}(0)=3 r_{\min }$ while circles relate to $R_{\text {pore }}(0)=4 r_{\text {min }}$.

transition between two or several states of a cluster, some of them (metastable) correspond to the cluster with a pore inside while the other (stable) to the cluster without pore. If suppose, that the probability of such transition, $\omega$, is independent on time, then pore life time $t_{\text {relax }} \sim \omega^{-1} \sim \exp (B / T)$, where $B$ is the height of the barrier between these states. Consequently, $B$ can be estimated as proportionality coefficient between $1 / T$ and $\ln t_{\text {relax }}$. In Fig. 4, temperature dependences of $t_{\text {relax }}$ are shown for $R_{\text {pore }}(0)$ $=4$ and $3 r_{\text {min }}$, that give following estimate of barrier heigth: $B\left(3 r_{m i n}\right) \sim 50 \mathrm{D}, B\left(3 r_{m i n}\right)$ $\sim 90$ D. Thus, pore dissolving process is connected with the collective transitions, that involve the number of atoms comparable with that of pore surface. It is worth to note that $T_{c}\left(R_{\text {pore }}(0), N\right)$ does not coincide with phase transition temperature between solid and liquid states, since it depends also on the initial pore size. Evidently, $T_{c}$ $\left(R_{\text {pore }}(0), N\right)$ is lower than phase transition temperature since nonequilbrium process leads to local heating of a cluster.

3.2. Relaxation of cluster with pore with initial temperature higher then $T_{c}$

As cluster temperature reaches values $T>T_{c}$ $\left(R_{\text {pore }}(0), N\right)$, the described above effect of pore volume stabilization vanishes. Here, like in the described above case, nonequilibrium process can either leave system in the initial phase state or make it transfer to the less ordered one (e.g. from state with solid core and liquid shell to the completely liquid state). As temperature grows, both in-

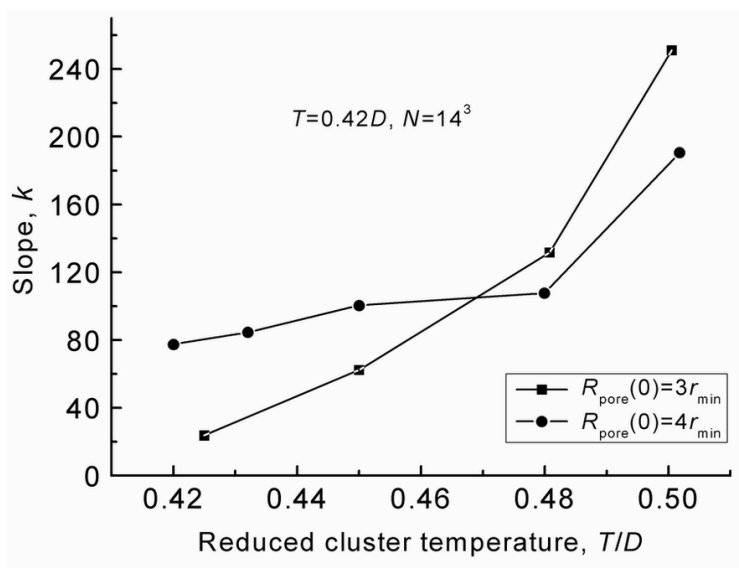

Fig. 5. Temperature dependences of slope of linear dependence of reduced pore volume on time for initial cluster conformations at temperature higher $T_{c}$, while heating cluster starting from solid state. Square denote the case of $R_{\text {pore }}(0)=3 r_{\text {min }}$ while circles relate to $R_{\text {pore }}(0)=4 r_{\text {min }}$.

itial and final (after relaxation) states of cluster become liquid. Pore relaxation time decreases sharply at $T>T_{c}$ (see Fig. 5). After fast initial decrease of $V_{\text {pore }}(t)$ by approximately $25 \%$ of its initial value, $V_{\text {pore }}$ decreases linearly with time. Slopes $k$ of these linear dependences as function of temperature for the case $N=14 R_{\text {pore }}=3 r_{\text {min }}$, $4 r_{\min }$ are shown in Fig. 5. Intersection point of these plots corresponds to the temperature at which initial cluster becomes completely liquid (most disordered state). Then cutting the pore does not cause any phase transfer and pores with larger radii exist longer than those with smaller ones since their dissolving requires larger outflow of vacancies.

\section{Conclusions}

Process of the relaxation of a cluster with an intrinsic pore is mainly determined by cluster initial phase state, that can be solid, liquid and solid with a liquid shell.

In case when initial cluster state is solid, for each pair of values $R_{\text {pore }}(0), N$, there exists critical temperature $T_{c}$, below which nonequilibrium process, initiated by instant cutting the pore off the cluster does not destroy solid state of the cluster, and pore, on average, exists longer than 12 ps. Process of pore relaxation in this case is characterized by a free energy barrier. $T_{c}$ grows monotonously with increasing cluster size and decreasing initial pore size. At temperatures higher $T_{c}$ pore relaxation process is 
sharply accelerated, and dependence of pore volume on time becomes linear.

\section{References}

1. P.G.Cheremskoj, V.V.Slezov, V.I.Betekhtin, Voids in solids, Moscow, Ehnergoatomizdat publ., 376 (1990) [in Russian].

2. B.M.Smirnov, Usp. Fiz. Nauk, 164, 1165 (1994)

3. B.M.Smirnov, Usp. Fiz.Nauk, 177, 369 (2007)
4. H.Reiss, H.L.Frisch, J.L.Lebowitz, J.Chem. Phys., 31, 369 (1959)

5. B.M.Smirnov, Clusters and Small Particles: in Gases and Plasmas, Springer-Verlag, New York (2000).

6. L.Verlet, Phys. Rev., 165, 201 (1967).

7. L.Verlet, Phys. Rev., 159, 98 (1967).

8. H.C.Andersen, J.Comput.Phys., 52, 24 (1983). 